\title{
Wolbachia plays no role in the one-way reproductive incompatibility between the hybridizing field crickets Gryllus firmus and G. pennsylvanicus
}

\author{
LS Maroja ${ }^{1}$, ME Clark $^{2}$ and RG Harrison ${ }^{1}$ \\ ${ }^{1}$ Department of Ecology and Evolutionary Biology, Cornell University, Ithaca, NY, USA and ${ }^{2}$ Department of Biology, University of \\ Rochester, Rochester, NY, USA
}

\begin{abstract}
Wolbachia are cytoplasmically inherited $\alpha$-proteobacteria that can cause cytoplasmic incompatibility $(\mathrm{Cl})$ in insects. This incompatibility between sperm and egg is evident when uninfected females mate with infected males. Wolbachiadriven reproductive incompatibilities are of special interest because they may play a role in speciation. However, the presence of Wolbachia does not always imply incompatibility. The field crickets Gryllus firmus and $G$. pennsylvanicus exhibit a very clear unidirectional incompatibility and have
\end{abstract}

been cited as a possible example of Wolbachia-induced $\mathrm{Cl}$. Here, we conduct curing experiments, intra- and interspecific crosses, cytological examination of Wolbachia in testes and Wolbachia quantifications through real-time PCR. All of our data strongly suggest that Wolbachia are not involved in the reproductive incompatibility between $G$. firmus and G. pennsylvanicus.

Heredity (2008) 101, 435-444; doi:10.1038/hdy.2008.75; published online 6 August 2008

Keywords: real-time PCR; reproductive isolation; cytoplasmic incompatibility; antibiotic treatment

\section{Introduction}

Wolbachia are cytoplasmically inherited $\alpha$-proteobacteria that can infect reproductive tissues of insects and cause reproductive alterations, including parthenogenesis (Stouthamer et al., 1993), feminization of males (Rousset et al., 1992), male killing (Hurst et al., 1999) and cytoplasmic incompatibility (CI) (Breeuwer et al., 1992; $\mathrm{O}^{\prime}$ Neill et al., 1992). Surveys have found that $16-76 \%$ of insects sampled are infected with Wolbachia (Werren et al., 1995; West et al., 1998; Jeyaprakash and Hoy, 2000; Werren and Windsor, 2000; Hilgenboecker et al., 2008). A recent meta-analysis adjusting for infection frequency within species predicts that around $66 \%$ of insects are infected with Wolbachia (Hilgenboecker et al., 2008). CI is a sperm-egg incompatibility, manifested when uninfected females mate with infected males. Shortly after fertilization, asynchrony in male and female pronuclei development leads to a series of mitotic defects and ultimately to embryonic death (Yen and Barr, 1971; Callaini et al., 1996; Lassy and Karr, 1996; Stouthamer et al., 1999; Tram and Sullivan, 2002). Although the molecular mechanism of CI is still poorly understood, it appears that Wolbachia present inside the testes 'modify' the sperm, which must then be 'rescued' in the egg by

Correspondence: Dr RG Harrison, Department of Ecology and Evolutionary Biology, Cornell University, Corson Hall, Ithaca, NY 14853, USA.

E-mail:rgh4@cornell.edu

Received 19 April 2008; revised 24 June 2008; accepted 5 July 2008; published online 6 August 2008 the same Wolbachia strain, if successful embryonic development is to occur (Werren, 1997a). Wolbachia strains can be classified based on their ability to modify sperm $\left(\mathrm{mod}^{+}\right.$or $\left.\bmod ^{-}\right)$and rescue in eggs (resc ${ }^{+}$and resc $^{-}$) (Werren, 1997a).

The effect of CI is unidirectional (usually involving crosses between infected and uninfected individuals) or bidirectional (involving crosses between individuals with different Wolbachia types) (Barr, 1980; Breeuwer and Werren, 1990; O'Neill and Karr, 1990). Wolbachiadriven reproductive incompatibilities are of special interest because they may play a role in speciation by facilitating the evolution of reproductive isolation between incipient species (Werren, 1997b; Telschow et al., 2005a, b; Jaenike et al., 2006).

The presence of Wolbachia does not always imply incompatibility. Wolbachia infections exist with no obvious phenotypic effects (Hoffmann et al., 1996). Even in cases where incompatibility is observed, Wolbachia may not be the causal agent (Weeks et al., 2002). Nuclear genes can also be involved, and in some cases both nuclear-induced and Wolbachia-induced incompatibilities are known to play a role (Breeuwer and Werren, 1995; Navajas et al., 2000; Vala et al., 2000). Proving that Wolbachia is a causal agent requires curing experiments (treatment with antibiotics) and a rigorous series of crosses between infected and uninfected individuals with the same genetic background.

The hybridizing field crickets Gryllus firmus and G. pennsylvanicus exhibit a one-way reproductive incompatibility; female G. firmus produce no progeny when mated with male $G$. pennsylvanicus, but the reciprocal 
cross produces viable and fertile offspring (Harrison, 1983). The bimodal mosaic hybrid zone formed by these very closely related species is a well-studied model system in speciation research (Harrison, 1983, 1985; Harrison and Rand, 1989; Willett et al., 1997; Ross and Harrison, 2002, 2006). Understanding the causes of the one-way incompatibility between G. firmus and G. pennsylvanicus is important for understanding the evolution of barriers to gene exchange.

Because Gryllus species harbor Wolbachia, it has been proposed that the bacterial infections are the cause of the one-way reproductive incompatibility (Giordano et al., 1997). Giordano et al. (1997) argued that G. pennsylvanicus was infected, whereas G. firmus was not, consistent with the pattern expected for Wolbachia-induced CI. However, due to incorrect assignment of crickets to species and lack of a perfect correlation between species and infection status, this conclusion was later rejected (Mandel et al., 2001). Mandel et al. (2001) showed that many G. firmus are infected, harboring what they termed the wG2 Wolbachia strain. Most G. pennsylvanicus harbor the wG1 strain, but some individuals were doubly infected ( $w \mathrm{G} 1$ and $w \mathrm{G} 2$ ), and a few carried only the $w \mathrm{G} 2$ strain. Extrapolating from these results, Mandel et al. (2001) suggested that about $13 \%$ of the heterospecific crosses should produce offspring; yet in dozens of crosses observed, not a single one yielded any progeny (Harrison, 1983; RG Harrison, unpublished data). They concluded that Wolbachia is unlikely to play a role in the one-way reproductive incompatibility between $G$. firmus and G. pennsylvanicus.

Here, we present additional data that argue against a role for Wolbachia in reproductive isolation between the cricket species. We conduct curing experiments and intra- and interspecific crosses, use microscopy to examine the presence/absence of Wolbachia in reproductive tissues and quantify Wolbachia loads in G. firmus and G. pennsylvanicus using real-time PCR. If Wolbachia is responsible for the observed reproductive incompatibility ('the Wolbachia hypothesis'), then uninfected (cured) male G. pennsylvanicus should be able to sire hybrid progeny when mated with G. firmus females. Furthermore, under the 'Wolbachia hypothesis,' Wolbachia should be present somewhere in testes (Boyle et al., 1993; Bressac and Rousset, 1993; Giordano et al., 1995; Bourtzis et al., 1996; Poinsot et al., 1998; Clark et al., 2002, 2003, 2008; Riparbelli et al., 2007), and hybrid eggs should be fertilized but later fail to develop (Callaini et al., 1996, 1997; Lassy and Karr, 1996; Tram and Sullivan, 2002; Duron and Weill, 2006). None of these predictions were supported, and we thus reiterate Mandel's et al. (2001) conclusion that Wolbachia infections are not the cause of the one-way reproductive incompatibility between G. firmus and G. pennsylvanicus.

\section{Materials and methods}

\section{Cricket rearing}

We collected late instar G. firmus nymphs in Guilford, CT, USA $\left(41^{\circ} 15^{\prime} ;-72^{\circ} 42^{\prime}\right)$ and $G$. pennsylvanicus nymphs in Ithaca, NY, USA $\left(42^{\circ} 24^{\prime} ;-76^{\circ} 31^{\prime}\right)$. Both species were collected during August-September 2004. We sorted the crickets by species and maintained five plastic cages $(30 \mathrm{~cm} \times 16 \mathrm{~cm} \times 9 \mathrm{~cm})$ for each species. Each cage con- tained five males and five females (total of 50 crickets for each species). Crickets were provided with ad libitum food (Purina Cat Chow, Nestle Purina, St Louis, MO, USA), a water vial, cardboard for shelter and a Petri dish of sterilized soil as oviposition substrate. The cages were kept at $25^{\circ} \mathrm{C}, 12 \mathrm{~h}-12 \mathrm{~h}$ light-dark. Oviposition dishes containing eggs were incubated for a maximum of 40 days at $25^{\circ} \mathrm{C}$ and then placed in a refrigerator at $4{ }^{\circ} \mathrm{C}$ for 102 days to insure synchronous hatch of nymphs (Harrison, 1985).

Hatching started on 15 February 2005, 21 days after eggs were removed from the refrigerator. We divided the offspring from each species into two groups: an antibiotic feeding treatment and an untreated control. Crickets from each group/species were reared in a separate plastic group cage $(65 \mathrm{~cm} \times 45 \mathrm{~cm} \times 40 \mathrm{~cm})$ with ad libitum food (Purina Cat Chow) and oviposition dishes. The antibiotic treatment group received $0.25 \%$ tetracycline $\mathrm{HCl}$ (Sigma, St Louis, MO, USA) in the water (changed three times per week) throughout development, whereas the untreated group received pure water. Individuals within each group were allowed to mate freely. Oviposition dishes were treated as described above and hatching began on 2 January 2006. Treatment was continued as in the previous generation. In March 2006, virgin late instar crickets were separated to single-sex plastic cages $(30 \mathrm{~cm} \times 16 \mathrm{~cm} \times 9 \mathrm{~cm})$, receiving the same treatment as before, until assigned to an experimental cross.

\section{Experimental crosses}

Our experimental design for crosses was the most complete possible given the poor performance of our $G$. pennsylvanicus colonies (only four untreated and six treated males were ultimately available for crossing). Each G. pennsylvanicus male was therefore mated with multiple G. firmus females. To discover whether Wolbachia causes intraspecific CI in G. firmus, we also conducted crosses between treated and untreated G. firmus.

We abbreviate cross types using three letters (for example, $\mathrm{H} / \mathrm{tu}$ ): The first letter indicates whether the cross is heterospecific or conspecific ( $\mathrm{H}$ or $\mathrm{C}$ ), the second letter indicates the male group (treated, $t$ or untreated, $u$ ) and the third letter indicates the female group ( $t$ or $\mathrm{u}$ ). Experimental females are always G. firmus, thus an $\mathrm{H} / \mathrm{tt}$ cross involves a treated $G$. pennsylvanicus male and a treated G. firmus female, whereas a C/tt cross involves a treated $G$. firmus male and female.

For the heterospecific crosses $(\mathrm{H})$, a treated $(\mathrm{t})$ or untreated (u) G. pennsylvanicus male was put with two treated (t) or untreated (u) G. firmus females for 3 days. Females were then removed to individual plastic cages and provided with ad libitum food, water and an oviposition dish. The male was placed in an individual cage and reused in subsequent matings. Twenty-six G. firmus females were crossed to six treated G. pennsylvanicus males: eight females were treated $(\mathrm{H} / \mathrm{tt})$ and 18 females were untreated $(\mathrm{H} / \mathrm{tu})$. Twentysix G. firmus females were crossed with four untreated males: 10 females were treated $(\mathrm{H} / \mathrm{ut})$ and 16 females were untreated $(\mathrm{H} / \mathrm{uu})$. All crosses resulted in spermatophore transfer to the female.

For the conspecific (C) crosses, a treated (t) or untreated $(\mathrm{u})$ G. firmus male was paired with a treated (t) or untreated $(\mathrm{u}) \mathrm{G}$. firmus female for 3 days. The male 
was then frozen at $-80^{\circ} \mathrm{C}$ for DNA extraction, and the female was placed in an individual plastic cage as described above. We performed 32 crosses using 16 treated and 16 untreated males: 5 males of each group mated with a treated female $(\mathrm{C} / \mathrm{tt}$ and $\mathrm{C} / \mathrm{ut})$ and 11 males of each group mated with an untreated female $(\mathrm{C} / \mathrm{tu}$ and $\mathrm{C} / \mathrm{uu})$. Again, all females were observed with a spermatophore.

All females used in the crosses were 7-10 days old. Female post-mating lifespan was estimated as days from mating until death. Lifetime fecundity was assessed by counting all eggs laid by each female. Eggs were separated from the oviposition substrate using a series of sieves and counted under a stereoscopic microscope.

Fecundity (number of eggs) and fertility (proportion of eggs hatching) for conspecific and heterospecific crosses were analyzed separately. Female lifespan data were analyzed combining data from conspecific and heterospecific crosses.

Data on conspecific fertility were fitted to a general linear model (GLM), weighting for fecundity. Conspecific fecundity and female lifespan data were fitted to a GLM with negative binomial errors. Residuals of all the performed GLMs were analyzed by visual inspection and no significant deviations from normality were observed. No outliers were found using Cook's statistics values. The effects of all dependent variables on the response variables were tested using log-likelihood ratio tests comparing the deviance of a model including and excluding the factor being tested. All analyses were performed with R. 2.6.1 ( $\mathrm{R}$ Development Core Team, 2006). All results are shown as mean \pm s.d., $n$.

\section{Real-time PCR}

To determine the Wolbachia load of each cricket, we used TaqMan real-time PCR. This approach measures the relative number of template molecules for a Wolbachiaspecific gene compared with template number for a cricket-specific gene (elongation factor, EF1 $\alpha$ ). We extracted DNA from whole abdomen of treated and untreated crickets using DNeasy tissue kit (Qiagen, Valencia, CA, USA). All DNA extractions were diluted to $10 \mu \mathrm{g} / \mu \mathrm{l}$.

Primers and TaqMan fluorescence-labeled probes for real-time PCR assays were designed using Primer Express Software (Applied Biosystems, Foster City, CA, USA). These primers specifically amplify Gryllus EF1 $\alpha$ (GenBank accession numbers, DQ630925 and DQ630927) and Wolbachia ftsZ (GenBank accession numbers, U28195 and
U83100, which correspond to the two types of Wolbachia found in G. firmus and G. pennsylvanicus; see Mandel et al. 2001). Primers and probes are shown in Table 1.

For real-time PCR assays, $2 \mu \mathrm{l}$ of the extracted genomic DNA template $(20 \mu \mathrm{g})$ was combined with $900 \mu \mathrm{M}$ of each oligonucleotide primer and $250 \mu \mathrm{M}$ of the TET-(EF1 $\alpha)$ or 6FAM-(ftsZ) and TAMRA-labeled probe in $25 \mu \mathrm{l}$ of total reaction volume using TaqMan Universal PCR Master Mix (Applied Biosystems). TaqMan PCR reactions were mixed in 96-well MicroAmp optical plates (Applied Biosystems). The PCR samples were subjected to 45 cycles of amplification in an ABI 7500 real-time PCR System (Applied Biosystems) under the following conditions: $50^{\circ} \mathrm{C}$ for 2 min (uracil $N$-deglycosylase digest), $95^{\circ} \mathrm{C}$ for $10 \mathrm{~min}$ (AmpliTaq Gold pre-activation) and then 40 cycles of $95^{\circ} \mathrm{C}$ for $15 \mathrm{~s}$ and $60^{\circ} \mathrm{C}$ for $1 \mathrm{~min}$. The fluorescence data were analyzed using the Applied Biosystems software. The standard curve was always prepared using a dilution series (up to $10^{-5}$ ) for the same DNA sample; water (in place of DNA) was used as blank. Each individual sample was assayed at least twice, but because differences in DNA concentration between replicates were always $<0.01 \times$ (after standardization-see below), we report only the average value for each individual.

Our real-time DNA measurements are relative not absolute; therefore, Wolbachia loads reported here are only meaningful for samples in this study. To calculate relative values, we assigned an arbitrary DNA quantity for each of the dilutions in the standard curve, from $10^{5}$ in the $1 \times$ dilution to 1 in the $10^{5} \times$ dilution. On the basis of the $C_{t}$ (threshold cycle) of each sample and the standard curve, we obtained estimates of EF1 $\alpha$ and $\mathrm{ftsZ}$ amounts for each individual. We then divided the amount of ftsZ DNA by the amount of EF1 $\alpha$ DNA, to generate a normalized value. Finally, a randomly selected untreated individual was chosen to represent a standard $1 \times$ Wolbachia load, and all other values were adjusted in relation to this standard.

We quantified Wolbachia load from a total of 53 crickets. These included parents from all conspecific crosses that failed to produce offspring $(\mathrm{C} / \mathrm{tt}, n=3 ; \mathrm{C} / \mathrm{tu}$, $n=3$; C/ut, $n=2)$ as well as from all crosses that produced offspring but were not expected to do so under the hypothesis of Wolbachia-induced CI (untreated male and treated female, $\mathrm{C} / \mathrm{ut}, n=3$ ). In addition, we quantified all G. pennsylvanicus males $(n=5$ for treated and $n=4$ for untreated) and G. pennsylvanicus females ( $n=3$ for treated and $n=3$ for untreated). We also included the following randomly chosen individuals: five G. firmus treated males, five G. firmus untreated

Table 1 Primers and probes for TaqMan real-time PCR for Gryllus and Wolbachia genes

\begin{tabular}{llll}
\hline Species & Primer or probe & Sequence $\left(5^{\prime}-3^{\prime}\right)$ & Position \\
\hline Gryllus... & EF1 $\alpha \_F$ & CTGACCTCCGCAGCAACA & $569-587$ \\
& EF1 $\alpha \_R$ & TTGCCAGTGGTCGAACACA & $612-630$ \\
& EF1 $\alpha$ probe $($ TET $)$ & TGGCCAGGCATTCCCTCAGT & $591-610$ \\
Wolbachia... & ftsZ_F & TGAAGAAGTGGATGAAAATGCAAA & $729-752$ \\
& ftsZ_R & GCCAGTTGCAAGAACAGAAACTC & $800-822$ \\
& ftsZ probe $(6 \mathrm{FAM})$ & ACTTTTGATCAGGCGATGGAGGGAAGA & $769-795$
\end{tabular}

The nucleotide positions are those reported in GenBank (accession number DQ630925 for EF1 $\alpha$ and U28195 for ftsZ). 
males, five G. firmus treated females and five G. firmus untreated females. Wolbachia loads in one male from an infertile cross $(\mathrm{C} / \mathrm{tu})$ and two males from $\mathrm{C} / \mathrm{ut}$ crosses, as well as one male G. pennsylvanicus, could not be measured due to poor quality or unavailable DNA.

We assayed an additional 10 wild-caught G. pennsylvanicus individuals from Ithaca, NY, USA (five males and five females) to compare Wolbachia loads of captive and wild individuals. These crickets were captured as late instar nymphs in August 2007.

To test for differences in Wolbachia loads between treatments, sexes and species, we fitted our data to GLMs with $\gamma$ errors using R. 2.6.1 (R Development Core Team, 2006) as explained above (experimental crosses section).

\section{RFLP analysis}

Mandel et al. (2001) reported two different Wolbachia strains, wG1 (accession number U83100) and wG2 (accession number U28195), common to G. firmus and G. pennsylvanicus. We used an RFLP analysis to determine the strain of Wolbachia for each individual assayed with RT-PCR. Universal primers to both strains, ftsZ12F (5'-AAAAATTCAACTTGGTATCAA-3') and ftsZ812R (5'-AGAACAGAAACTCTAACTCTTCC-3'), were used to amplify a short fragment of Wolbachia-specific ftsZ. These amplifications were carried out in $10 \mu \mathrm{l}$ PCR reactions and contained $3 \mathrm{mM} \mathrm{MgCl}_{2}, 0.2 \mathrm{mM} \mathrm{dNTPs}$, $50 \mathrm{mM} \mathrm{KCl}, 20 \mathrm{mM}$ Tris ( $\mathrm{pH} 8.4$ ), $2.5 \mu \mathrm{g}$ of each primer, $1 \mathrm{U}$ of platinum Taq DNA polymerase (Invitrogen, Carlsbad, CA, USA) and $1 \mu \mathrm{l}$ DNA $(20-30 \mu \mathrm{g})$. Conditions for thermal cycling were 2 min at $95^{\circ} \mathrm{C}$ followed by 35 cycles of $50 \mathrm{~s}$ at $94{ }^{\circ} \mathrm{C}, 60 \mathrm{~s}$ at $55^{\circ} \mathrm{C}$ and $90 \mathrm{~s}$ at $72^{\circ} \mathrm{C}$. The resulting $800-\mathrm{bp} \mathrm{fts} Z$ fragment is differentially cut by the restriction enzyme $A l u \mathrm{I}$. We fully digested $3-5 \mu \mathrm{l}$ of PCR product with $A l u \mathrm{I}$ and ran the digests on $2 \%$ agarose gels, using a doubly infected individual as a control on each gel. We could unambiguously assign Wolbachia strain(s) for all assayed infected individuals.

\section{Microscopy}

Gryllus testes and ovaries were dissected from adults in a small Petri dish with Tris-buffered saline Tween-20 (TBST) (50 mM Tris, $150 \mathrm{mM} \mathrm{NaCl}, 0.1 \%$ Tween, $0.05 \%$ $\mathrm{NaN}_{3}, \mathrm{pH}$ 7.5). Whole testes and ovaries were removed and transferred to a depression slide with TBST. Individual follicles were removed from testes, and ovarioles were separated and transferred to $3.7 \%$ formaldehyde in TBST for 15-30 min followed by three washes in TBST. Tissues were blocked in TBST with $1 \%$ bovine serum albumin for $10 \mathrm{~min}$. Wolbachia was visualized using an anti-human hsp60 mouse monoclonal antibody (Sigma), which recognizes Wolbachia (Hoerauf et al., 2000; McGraw et al., 2002). Tissues were incubated in the primary antibody solution (1:500 in TBST, $1 \%$ bovine serum albumin, $2 \mathrm{mg} / \mathrm{ml}$ RNaseA and 1:500 of the anti-wsp antibody) for $1 \mathrm{~h}$ at room temperature, followed by three washes with TBST. This was followed by $1 \mathrm{~h}$ at room temperature in 1:500 Alexa-Fluor 488 antimouse antibody (Molecular Probes), followed by three washes in TBST. DNA was then stained with either $1 \mu \mathrm{g} /$ $\mathrm{ml}$ DAPI (Invitrogen) for $5 \mathrm{~min}$ or with $5 \mu \mathrm{g} / \mathrm{ml}$ propidium iodide (Invitrogen) for $20 \mathrm{~min}$, followed by a brief wash in TBST before mounting in ProLong Gold antifade mounting media (Invitrogen). Images were obtained using a Zeiss Axio-Imager Z1 microscope. We observed both captive and wild-caught untreated crickets as well as first and second generation antibiotictreated crickets.

\section{Egg analysis}

Anecdotal evidence indicates that eggs from hybrid crosses (G. firmus female $\times G$. pennsylvanicus) are smaller and resemble unfertilized eggs. By measuring eggs to the nearest $0.01 \mathrm{~mm}$ under a dissecting scope ( $\times 15$ magnification), we obtained data on length and width of 10 unfertilized G. firmus eggs (virgin females), 10 eggs from crosses within G. firmus and 10 eggs from heterospecific crosses. All eggs were from untreated individuals and were measured during the diapause period (after 1 month at $4{ }^{\circ} \mathrm{C}$ ).

We also extracted DNA from individual eggs (six unfertilized, six pure G. firmus eggs and six eggs from crosses between $G$. firmus females and $G$. pennsylvanicus males) using a forensic QIAamp DNA micro kit (final dilution in $20 \mu \mathrm{l}$ ). All eggs were from untreated females, and DNA was extracted when eggs were in diapause (after 1 month at $4{ }^{\circ} \mathrm{C}$ ). After estimating the amount of DNA in each sample, we used cricket-specific microsatellites to test for the presence of maternal and paternal alleles. We also used Wolbachia ftsZ primers and cricketspecific EF1 $\alpha$ primers (as a positive control). PCRs $(10 \mu \mathrm{l}$ volume) contained $3 \mathrm{mM} \mathrm{MgCl}, 0.2 \mathrm{mM}$ dNTPs, $50 \mathrm{mM}$ $\mathrm{KCl}, 20 \mathrm{mM}$ Tris ( $\mathrm{pH} 8.4$ ), $2.5 \mu \mathrm{g}$ of each primer, $1 \mathrm{U}$ of platinum Taq DNA polymerase (Invitrogen) and $2 \mu \mathrm{l}$ DNA (20-30 $\mu \mathrm{g})$. PCR amplifications were performed using a thermal cycler (OmniGene, Hybaid) under the following conditions: 40 cycles of $50 \mathrm{~s}$ at $94{ }^{\circ} \mathrm{C}, 60 \mathrm{~s}$ at $52^{\circ} \mathrm{C}$ and $90 \mathrm{~s}$ at $72{ }^{\circ} \mathrm{C}$.

\section{Results}

\section{Experimental crosses}

In heterospecific crosses ( $G$. pennsylvanicus male $\times$ G. firmus female), antibiotic treatment had no effect on whether offspring were produced. All of the heterospecific crosses, except for one, failed to produce offspring, including those expected to produce offspring under the assumption that CI-inducing Wolbachia had been eliminated (Table 2). The single hybrid produced (the first ever reported in thousands of hybrid cross observations) came from an $\mathrm{H} / \mathrm{uu}$ cross, in which both parents were infected as shown by RT-PCR analysis (see below). The fecundity of heterospecific crosses was low $(67.9 \pm 92.2$ eggs, $n=52)$.

On average, conspecific crosses had higher fecundity (373 \pm 212 eggs, $n=32)$ and fertility (68.9 \pm 67.9 offspring, $n=32)$ than heterospecific crosses $(\mathrm{F}(1,84)=76$, $P=0.0001$ and $\mathrm{F}(1,84)=51.6, P<0.0001$, respectively), although some females from conspecific crosses (primarily from the treated group) failed to produce any offspring (Table 2). The conspecific crosses showed no significant male treatment $\times$ female treatment interaction in fecundity, and female treatment alone had a marginally nonsignificant effect $(\mathrm{F}(29,1)=69, P=0.09)$-treated females appear to deposit fewer eggs than untreated females $(272 \pm 244$ eggs, $n=10$ vs $419 \pm 183$ eggs, $n=22$, respectively). Antibiotic treatment had no significant effect on the percentage of eggs hatching. If Wolbachia 
Table 2 Results of experimental crosses

\begin{tabular}{|c|c|c|c|c|c|c|c|c|c|}
\hline & Cross type & Fertility exp? & Mean eggs & s.d. eggs & Mean offsprings & s.d. offsprings & (n) total & (n) fecund & (n) fertile \\
\hline \multirow[t]{4}{*}{ Conspecific } & $\mathrm{C} / \mathrm{uu}$ & Yes & 468.8 & 159.2 & 80.2 & 56.4 & 11 & 11 & 11 \\
\hline & $\mathrm{C} / \mathrm{tu}$ & Yes & 369.4 & 199.1 & 69.4 & 76.5 & 11 & 11 & 8 \\
\hline & $\mathrm{C} / \mathrm{tt}$ & Yes & 293.2 & 319.1 & 42.6 & 58.4 & 5 & 5 & 2 \\
\hline & $\mathrm{C} /$ ut & No & 250.2 & 176.4 & 69.2 & 92.6 & 5 & 5 & 3 \\
\hline \multirow[t]{4}{*}{ Heterospecific } & $\mathrm{H} / \mathrm{uu}$ & No & 86.2 & 115.2 & $0.06^{\mathrm{a}}$ & 0.25 & 16 & 15 & 1 \\
\hline & $\mathrm{H} / \mathrm{tu}$ & Yes & 64.4 & 78.1 & 0 & 0 & 18 & 16 & 0 \\
\hline & $\mathrm{H} / \mathrm{tt}$ & Yes & 66.5 & 77.3 & 0 & 0 & 8 & 8 & 0 \\
\hline & $\mathrm{H} / \mathrm{ut}$ & No & 46.4 & 93.5 & 0 & 0 & 10 & 8 & 0 \\
\hline
\end{tabular}

ane hybrid offspring was produced in this cross type. Both parents were infected (RT-PCR and RFLP results).

'Fertility exp?' indicates whether fertility is expected under the Wolbachia hypothesis. Results are fecundity (mean eggs and standard deviation), fertility (mean number of offspring and standard deviation), total number of females in each cross type ( $n$ total), number of fecund females ( $n$ fecund) and number of fertile females ( $n$ fertile). For cross-type abbreviations, see Materials and methods.

caused a reproductive incompatibility within species, the $\mathrm{C} /$ ut cross should fail to produce offspring (assuming that females were cured); yet this cross was equally fertile.

Antibiotic treatment decreased female post-mating lifespan $(\mathrm{F}(80,1)=140.5, \quad P<0.0001)$; treated females lived for $29.4 \pm 14.6$ days $(n=28)$, whereas untreated females lived for $43.7 \pm 14.5$ days $(n=56)$. Unexpectedly, mating with conspecifics or heterospecifics also affected female lifespan $(\mathrm{F}(82,1)=167.6, P<0.0001)$. Females mated to conspecifics lived longer than females mated to heterospecifics ( $45.8 \pm 14.6$ days, $n=32$ vs $35.7 \pm 15.4$ days, $n=52$, respectively).

\section{Real-time PCR}

Individual Wolbachia loads ranged from $0 \times$ to $172 \times$. G. pennsylvanicus had a significantly lower Wolbachia load than G. firmus $(\mathrm{F}(1,51)=44.6, P<0.001)$ and females had significantly higher loads than males in both species $(\mathrm{F}(1,50)=45.19, P<0.03)$. In addition, males responded better to treatment than females (significant sex $\times$ treatment interaction $(\mathrm{F}(1,46)=5.37, P<0.03$; Table 3). Furthermore, laboratory-reared G. pennsylvanicus had significantly lower Wolbachia loads than their wild counterparts $(\mathrm{F}(1,23)=45.51, P<0.001$; Table 3$)$. Because of the high variance in load between treatments/ individuals/species and the presence of cured individuals, it is very unlikely that we were quantifying Wolbachia DNA that had been transferred to the host genome.

In spite of two generations of antibiotic treatment, only 9 of the 53 assayed individuals showed no evidence of Wolbachia infection; 8 of these crickets were treated and 1 was an untreated G. pennsylvanicus female. Of the nine cured individuals, two were G. pennsylvanicus (one male and one female) and seven were G. firmus (three males and four females). The cured G. pennsylvanicus male was mated with four $G$. firmus females, none of whom produced any offspring. Only one of the cured G. firmus female was in a $\mathrm{C} /$ ut cross; she mated with a heavily infected male $(46 \times$ load) and produced 30 offspring (19\% hatching success). The other three cured G. firmus females were in the $\mathrm{C} / \mathrm{tt}$ group and these females failed to produce offspring. Two of these cured females were paired with two of the cured males $(0 \times$ load $)$ and deposited only 1-2 eggs. The third cured female mated
Table 3 Wolbachia loads (relative to a standard individual) in Gryllus firmus and G. pennsylvanicus (mean, standard deviation and number of samples) for treated ( $\mathrm{t}$ ) and untreated $(\mathrm{u})$ males and females

\begin{tabular}{llrllllll}
\hline Species & Group & \multicolumn{3}{c}{ Male } & & \multicolumn{3}{c}{ Female } \\
\cline { 3 - 4 } & & Mean & s.d. & $\mathrm{n}$ & & Mean & s.d. & $\mathrm{n}$ \\
\hline G. firmus & $\mathrm{t}$ & 4.2 & \pm 7.3 & 9 & & 35.8 & \pm 57.6 & 13 \\
& $\mathrm{u}$ & 22.9 & \pm 38.0 & 8 & & 39.6 & \pm 26.5 & 8 \\
G. pennsylvanicus & $\mathrm{t}$ & 0.3 & \pm 0.2 & 5 & & 0.8 & \pm 0.2 & 3 \\
& $\mathrm{u}$ & 0.4 & \pm 0.2 & 4 & & 0.8 & \pm 1.1 & 3 \\
& Wild & 5.8 & \pm 5.9 & 5 & & 7.5 & \pm 9.6 & 5 \\
\hline
\end{tabular}

with an infected male $(0.3 \times$ load $)$ and laid 301 eggs, all of which failed to hatch. These three $\mathrm{C} / \mathrm{tt}$ cured females had very reduced post-mating lifespan: 10 and 11 days for females mated with cured males and 27 days for the females mated with the infected males (compared to 46 days average for females mated with conspecifics). The third cured $G$. firmus male mated with an untreated female $(\mathrm{C} / \mathrm{tu})$ and produced only 12 offspring $(3.8 \%$ hatching success). The only hybrid offspring (identity confirmed with microsatellites) came from an $\mathrm{H} / \mathrm{uu}$ cross in which both male and female were infected $(0.3 \times$ and $16 \times$, respectively $)$.

\section{RFLP analysis}

We could assign a Wolbachia strain to all infected individuals. All experimental G. pennsylvanicus had the $w$ G1 strain $(n=13)$ and all experimental G. firmus had the $w \mathrm{G} 2$ strain $(n=31)$. However, 5 out of 10 wild-caught G. pennsylvanicus individuals had the wG2 type and only 3 had the $w \mathrm{G} 1$ strain ( 2 females were uninfected). There were no doubly infected individuals in either experimental or wild groups. An additional sample of 14 G. pennsylvanicus from three 'pure' populations in New York and Pennsylvania included 9 infected with wG1, 4 infected with wG2 and 1 doubly infected.

In the wild-caught Ithaca G. pennsylvanicus, there seems to be a difference in RT-PCR load between wG1 and wG2 Wolbachia strains. The average load for $w \mathrm{G} 1$ was $1.8 \times( \pm 1.2, n=3)$ and the average for $w \mathrm{G} 2$ was $12.2 \times( \pm 7.0, n=5)$. Because of the very small sample size we did not conduct any statistical tests. 


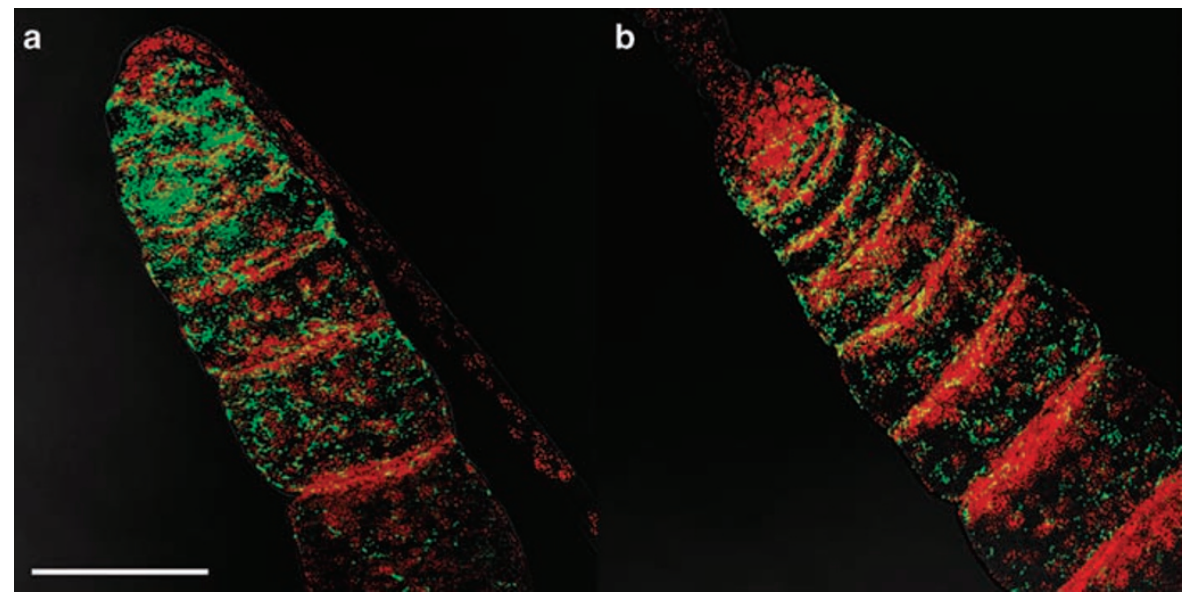

Figure 1 Wolbachia within developing ovaries of (a) Gryllus firmus and (b) G. pennsylvanicus. Host nuclei are stained with propidium iodide (red) and Wolbachia labeled with anti-hsp60 (green/yellow). Scale bar $=100 \mu \mathrm{m}$.

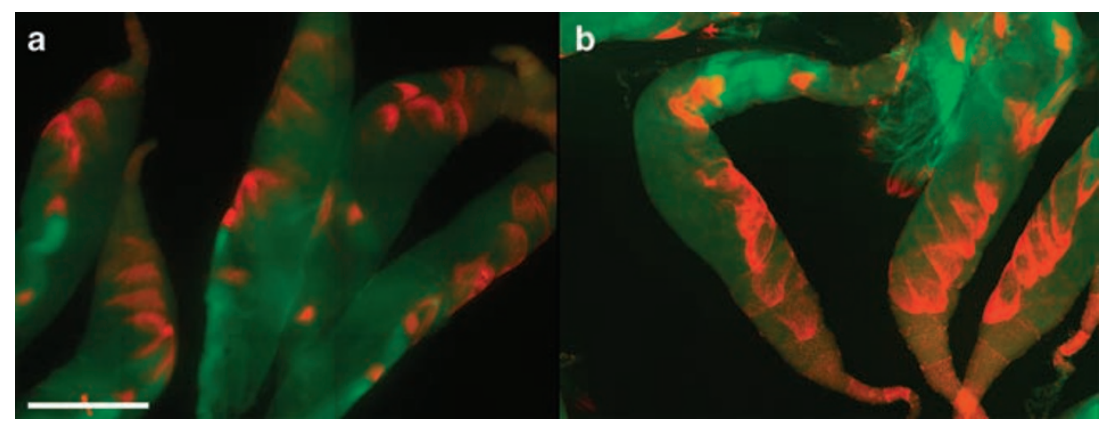

Figure 2 Wolbachia are absent from testis follicles in both Gryllus firmus (a) and G. pennsylvanicus (b). Spermatid nuclei (red) are seen within developing spermatocysts. All Alexa Fluor 488 staining (green) represents either background staining, or cross-reactivity with spermatid tails. Scale bar $=500 \mu \mathrm{m}$.

\section{Microscopy}

Wolbachia was easily visualized in ovaries from both G. firmus and G. pennsylvanicus (Figure 1). The overall Wolbachia load within ovaries is consistent with the RTPCR results, with higher Wolbachia densities within the ovaries of G. firmus than G. pennsylvanicus females. Within testes, Wolbachia was typically absent from both species (Figure 2) for treated, untreated and wild individuals. Wolbachia were never seen within the developing spermatocytes, spermatids or surrounding cyst cells. Very rarely, Wolbachia could be seen within a single somatic cell (not shown) in the outer follicle epithelium, but this was atypical.

\section{Egg analyses}

We used a one-way analysis of variance to test for differences between the mean length and width of unfertilized, hybrid cross and pure fertilized G. firmus eggs (see Figure 3). We found no significant difference in mean egg length (unfertilized $=3.11 \mathrm{~mm}$, hybrid cross $=3.08 \mathrm{~mm}$ and fertilized G. firmus $=3.13 \mathrm{~mm}$; $\mathrm{F}(2,27)=0.37, P>0.5)$, but a significant difference in the mean egg width (unfertilized $=0.631 \mathrm{~mm}$, hybrid cross $=0.641 \mathrm{~mm}$, and fertilized G. firmus $=0.953 \mathrm{~mm}$; $\mathrm{F}(2,27)=119.7, P<0.001)$. The post hoc Tukey test showed

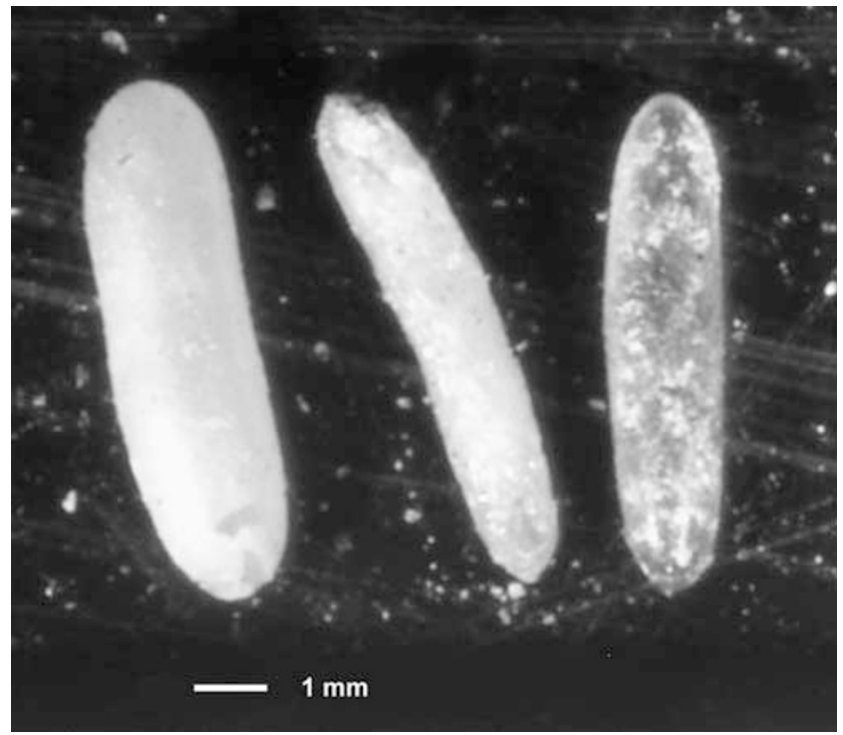

Figure 3 Eggs from Gryllus firmus females. The first is a typical fertilized G. firmus egg from a conspecific cross, the second is an unfertilized egg and the third is an egg from a hybrid cross. Hybrid cross eggs and unfertilized eggs are always narrower than a fertilized G. firmus egg. Color variation is commonly observed in unfertilized and hybrid cross eggs. 
that this difference was between fertilized G. firmus eggs and the other two categories. There were no differences between hybrid cross and unfertilized eggs $((U=H) \neq G f)$.

All egg DNA extractions had measurable amounts of nucleic acids (mean unfertilized $=11 \mu \mathrm{g} / \mu \mathrm{l}$, mean hybrid $=12 \mu \mathrm{g} / \mu \mathrm{l}$ and mean fertilized G. firmus $=18 \mu \mathrm{g} /$ $\mu 1)$; however, these measurements probably reflect carrier RNA added during the extractions. We were able to amplify Gryllus-specific microsatellites and Wolbachia ftsZ from all six eggs from crosses between G. firmus males and females. We also amplified Wolbachia ftsZ from three of six unfertilized eggs and four of six hybrid cross eggs; however, we were unable to amplify cricketspecific genes (microsatellites or EF1 $\alpha$ ) from any of the hybrid cross or unfertilized eggs.

\section{Discussion}

Overall, we found no evidence that Wolbachia infections play a role in the G. firmus/G. pennsylvanicus one-way reproductive incompatibility. The original observation (Giordano et al., 1997) that G. pennsylvanicus is infected and $G$. firmus is not infected is not supported by our data. However, our results do agree with the data of Mandel et al. (2001), showing that most individuals in northern populations of both species are infected, that both species harbor strains $w \mathrm{G} 1$ and $w \mathrm{G} 2$, but with somewhat different frequencies. Our results show that a completely cured G. pennsylvanicus male did not produce offspring when mated with G. firmus females, as would be expected if Wolbachia was the cause of reproductive incompatibility. Furthermore, we did not find Wolbachia inside the testes of adult males, although bacteria were present in other tissues. Finally, there is no evidence that eggs from hybrid crosses are fertilized and die later in development as would be expected if Wolbachia was responsible for the reproductive incompatibility. Wolbachia also does not seem to cause intraspecific $\mathrm{CI}$ in crickets; crosses expected to be incompatible ( $\mathrm{C} / \mathrm{ut}$ untreated males and treated females) produced as many offspring as controls, and a completely cured female was able to produce offspring with a highly infected male. Taken together, these observations provide strong evidence against the hypothesis of Wolbachia-induced CI in the field cricket hybrid zone.

\section{Interspecific one-way reproductive incompatibility}

Bacterial density is associated with the prevalence of Wolbachia-induced phenotypes, including the expression of CI. Treatments that reduce bacterial densities usually lead to decreases in Wolbachia-induced phenotypes (Breeuwer and Werren, 1993; Hurst et al., 2000; Zchori-Fein et al., 2000). Thus, if Wolbachia was the cause of the observed reproductive incompatibility, we would expect a decrease in CI with antibiotic treatment. Although treatment was not $100 \%$ effective, males had substantially reduced Wolbachia loads, and one G. pennsylvanicus male was completely cured (other treated G. pennsylvanicus males had loads $<0.5 \times$; Table 3). Despite the overall decrease in Wolbachia loads (especially in relation to field-collected individuals) and the successful cure, no hybrid crosses produced offspring (except for a single hybrid produced from an infected male and female). Moreover, given that higher bacterial densities are associated with increase in incompatibility and possibly higher efficacy in sperm modification and egg rescue (Boyle et al., 1993; Breeuwer and Werren, 1993; Bressac and Rousset, 1993; Poinsot et al., 1998; Stouthamer et al., 1999), if Wolbachia were the cause of reproductive incompatibility, we would expect higher bacterial loads in G. pennsylvanicus (as it should be the species with modified sperm). In contrast to those expectations, G. firmus had bacterial loads about $50 \times$ those in $G$. pennsylvanicus.

In Drosophila with CI, both bacterial density within testes (Boyle et al., 1993; Bressac and Rousset, 1993; Giordano et al., 1995; Bourtzis et al., 1996; Poinsot et al., 1998; Riparbelli et al., 2007) and total amount of infected germ cell cysts (Clark et al., 2002, 2003) have been implicated in CI expression. In other hosts, Wolbachia modify sperm without being present within an individual spermatocyte or spermatid during development, but instead are present in other cells within testes, suggesting that Wolbachia can either act across cells or act prior to spermatogenesis (Clark et al. 2008). Within the testes, CI-inducing $\left(\bmod ^{+}\right)$Wolbachia modify sperm, such that normal embryonic development can occur only if the modification is rescued by an infected (resc ${ }^{+}$) egg (Werren, 1997a). Wolbachia strains that neither modify nor rescue sperm $\left(\bmod ^{-}\right.$and resc $\left.^{-}\right)$also exist; these strains have no effect on host reproduction (Hoffmann et al., 1996; Veneti et al., 2003; Marshall, 2004). We did not observe Wolbachia inside testes or in the surrounding tissues (Figure 2). This absence of Wolbachia inside the testes of both Gryllus species suggests that these Wolbachia strains are unable to modify sperm and cause CI in these hosts.

CI is expected between hybridizing species when one species is infected with CI-causing Wolbachia strain and the other species is uninfected or lacks any of the Wolbachia types found in the other species. In our experimental crosses, we did not find any doubly infected individuals; all G. firmus were infected with the $w \mathrm{G} 2$ strain and all G. pennsylvanicus carried the $w \mathrm{G} 1$ strain. However, wild-caught G. pennsylvanicus carry either $w \mathrm{G} 1$ or $w \mathrm{G} 2$, both strains or are uninfected. On the basis of our observed ratios of wG1-infected, wG2infected and doubly infected, if Wolbachia were the primary cause of the incompatibility between $G$. firmus and G. pennsylvanicus, then a large fraction of heterospecific crosses should produce offspring. Contrary to this expectation, in hundreds of heterospecific crosses (Harrison, 1983; Maroja, 2008; RG Harrison, unpublished) only one hybrid has ever been produced (reported in this study). This hybrid came from a cross between untreated crickets, in which the G. pennsylvanicus male carried wG1 $(0.3 \times$ load $)$ and G. firmus female carried $w \mathrm{G} 2(16 \times$ load $)$. The extreme rarity of hybrids from $G$. firmus females suggests the existence of very strong barriers to fertilization. This is corroborated by the observation that mitochondrial DNA introgression across the cricket hybrid zone is always from G. pennsylvanicus into G. firmus (Harrison et al., 1987; Harrison and Bogdanowicz, 1997; Ross and Harrison, 2002) as would be expected if the only F1 hybrids produced were offspring of G. pennsylvanicus females.

Wolbachia-induced CI usually leads to early embryonic death or haploid development (Callaini et al., 1996, 1997; Lassy and Karr, 1996; Tram and Sullivan, 2002; Duron and Weill, 2006). However, cricket eggs from hybrid crosses resemble unfertilized eggs, both in size (Figure 3) 
and in the failure to provide suitable DNA templates for amplification of Gryllus-specific microsatellites, although still providing templates for amplification of Wolbachiaspecific genes. If there were early embryos in the eggs from heterospecific crosses, then the amount of DNA should be sufficient to allow amplification of Gryllusspecific microsatellites. It is possible that the DNA has degraded subsequent to the death of the embryo; however, in that case we would not expect to be able to amplify Wolbachia DNA.

\section{Wolbachia in conspecific crosses and antibiotic treatment effects}

Wolbachia does not appear to cause intraspecific CI in G. firmus. Wolbachia is not found in testes of G. firmus males (Figure 2) and is thus unlikely to modify sperm. Furthermore, our crossing data suggest that the observed infertility (or low fecundity) of some male-female pairs is a result of the antibiotic treatment itself, rather than due to presence or absence of Wolbachia. If Wolbachia caused conspecific CI, and if curing were complete, our $\mathrm{C} /$ ut crosses $(n=5)$ should be infertile as an uninfected egg would not be able to rescue modified sperm. The only completely cured female was mated with a highly infected male $(46 \times$ load) and had normal fertility. Two $\mathrm{C} /$ ut crosses also showed normal fertility (females had reduced Wolbachia loads of $5 \times$ and $3 \times$ ). Antibiotic treatment significantly reduces female fecundity independent of the status of the male and probably also explains the infertility of the other two C/ut crosses, as well as other infertile crosses (all of which involved treated individuals). In addition, antibiotic treatment decreased female post-mating lifespan. Taken together, these observations suggest direct negative effects of antibiotic treatment, effects that confound interpretation of curing experiments in these insects. Alternatively, Wolbachia could be a mutualist (Hoerauf et al., 1999; Dedeine et al., 2001; Weeks et al., 2007) and, in this case, the adverse effects on treated females would be due to a decrease in Wolbachia loads.

Curiously, we also found that mating with conspecific males vs heterospecific males affected female lifespan; females mated with conspecifics lived longer than females mated with heterospecifics. This unexpected result could be related to the benefits field cricket females appear to gain from conspecific matings, or an additional unappreciated cost to heterospecific matings (Simmons, 1988; Burpee and Sakaluk, 1993; Wagner et al., 2001; Sakaluk et al., 2002; Ivy and Sakaluk, 2005). Both costs and benefits might be exaggerated in captive/treated populations. Alternatively, observations of female lifespan differences might be a negative consequence of reduced oviposition rate and accumulation of eggs in females mated with heterospecific males.

\section{Wolbachia load}

Total Wolbachia loads were consistently much lower in males than in females in both species. This may reflect high Wolbachia densities in the female germline and may explain the persistence of Wolbachia in the absence of CI. High Wolbachia densities in the female germline likely ensure high rates of Wolbachia transmission. Interestingly, both wild-caught male and female G. pennsylvanicus had much higher Wolbachia loads than crickets reared in the lab. The basis of this difference is unclear. Previous reports from Drosophila suggest that the phenotypic effects of Wolbachia are greater under ideal laboratory conditions compared with either stressed laboratory conditions or wild-caught flies (Ikeda, 1970; Hurst et al., 2001). If lab conditions are non-optimal for G. pennsylvanicus, Wolbachia loads may be higher in individuals living in more optimal (wild) conditions compared with the suboptimal (laboratory) conditions.

The data presented here suggest that Wolbachia does not cause $C I$ in $G$. pennsylvanicus and does not currently play a role in the incompatibility between G. pennsylvanicus and G. firmus. Although Wolbachia infections have now been described in hundreds of arthropod species, the phenotypic effects of Wolbachia infection have been experimentally examined in only a few model organisms. These are restricted to species easily reared in a laboratory environment. A more thorough understanding of effects of Wolbachia on hosts will require examination of a wider range of hosts that may not be as amendable to a laboratory environment as traditional model organisms.

\section{Conclusions}

G. firmus and G. pennsylvanicus are an important model system in the study of speciation, and understanding the basis of their reproductive isolation is important for understanding the evolution of barriers to gene exchange. The importance of 'infectious speciation' (Coyne, 1992) caused by Wolbachia bidirectional CI is a subject of current debate (Werren, 1997b; Coyne and Orr, 2004). There is good evidence that Wolbachia infections do play a role in barriers to current gene exchange between species (Nasonia, Breeuwer and Werren, 1993; Bordenstein et al., 2001; Drosophila, Shoemaker et al., 1999; Jaenike et al., 2006). Although genetic incompatibilities are also involved in these examples, Wolbachia-driven incompatibility seems to have evolved early enough to have played a role in the initial speciation process. However, in these cases, as in virtually all studies of current barriers to gene exchange, it is very difficult to identify the order in which barriers have arisen (Bordenstein, 2003). As a result, the extent to which endosymbionts are important in insect speciation remains unclear. The field crickets G. firmus and G. pennsylvanicus exhibit a very clear unidirectional incompatibility and have been cited as a possible example of Wolbachiainduced CI. The data presented here strongly suggest that this is not the case.

\section{Acknowledgements}

We thank Rodman G Getchell for helping with the RT-PCR, Steve Bogdanowicz for technical advice in all aspects of this study and Jose A Andres for statistical advice. This project was supported by NSF grant DEB0639904 to RGH. The work of MEC was supported by NSF grant EF-0328363 to J Werren.

\section{References}

Barr AR (1980). Cytoplasmic incompatibility in natural populations of a mosquito, Culex pipiens. Nature 283: 71-72.

Bordenstein SR (2003). Symbiosis and the origin of species. In: Miller T, Bourtzis K (eds). Insect Symbiosis. CRC Press: New York. 
Bordenstein SR, O'Hara FP, Werren JH (2001). Wolbachiainduced incompatibility precedes other hybrid incompatibilities in Nasonia. Science 409: 675-677.

Bourtzis K, Nirgianaki A, Markakis G, Savakis C (1996). Wolbachia infection and cytoplasmic incompatibility in Drosophila species. Genetics 144: 1063-1073.

Boyle L, O'Neill SL, Robertson HM, Karr TL (1993). Interspecific and intraspecific horizontal transfer of Wolbachia in Drosophila. Science 260: 1796-1799.

Breeuwer JAJ, Stouthamer R, Barns SM, Pelletier DA, Weisburg WG, Werren JH (1992). Phylogeny of cytoplasmic incompatibility microorganisms in the parasitoid wasp genus Nasonia based on 16S ribosomal DNA sequences. Insect Mol Biol 1: 25-36.

Breeuwer JAJ, Werren JH (1990). Microorganisms associated with chromosome destruction and reproductive isolation between two insect species. Nature 346: 558-560.

Breeuwer JAJ, Werren JH (1993). Cytoplasmic incompatibility and bacterial density in Nasonia vitripennis. Genetics 135: 565-574.

Breeuwer JAJ, Werren JH (1995). Hybrid breakdown between two haploid species: the role of nuclear and cytoplasmic genes. Evolution 49: 705-717.

Bressac C, Rousset F (1993). The reproductive incompatibility system in Drosophila simulans: DAPI-staining analysis of the Wolbachia symbionts in sperm cysts. I Invert Pathol 61: 226-230.

Burpee DM, Sakaluk SK (1993). Repeated matings offset the costs of reproduction in female field crickets. Evol Ecol 7: 240-250.

Callaini G, Dallai R, Riparbelli MG (1997). Wolbachia-induced delay of paternal chromatin condensation does not prevent maternal chromosomes from entering anaphase in incompatible crosses of Drosophila simulans. J Cell Sci 110: 271-280.

Callaini GM, Riparbelli G, Giordano R, Dallai R (1996). Mitotic defects associated with cytoplasmic incompatibility in Drosophila simulans. I Invert Pathol 67: 55-64.

Clark ME, Bailey-Jourdain C, Ferree PM, England S, Sullivan W, Windsor D et al. (2008). Wolbachia modification of sperm does not always require residence within developing sperm. Heredity (in press).

Clark ME, Veneti Z, Bourtzis K, Karr TL (2002). The distribution and proliferation of the intracellular bacteria Wolbachia during spermatogenesis in Drosophila. Mech Dev 111: 3-15.

Clark ME, Veneti Z, Bourtzis K, Karr TL (2003). Wolbachia distribution and cytoplasmic incompatibility during sperm development: the cyst as the basic cellular unit of $\mathrm{CI}$ expression. Mech Dev 120: 185-198.

Coyne JA (1992). Genetics and speciation. Nature 355: 511-515.

Coyne JA, Orr HA (2004). Speciation. Sinauer Associates: Sunderland, MA.

Dedeine F, Vavre F, Fleury F, Loppin B, Hochberg ME, Boulétreau M (2001). Removing symbiotic Wolbachia bacteria specifically inhibits oogenesis in a parasitic wasp. Proc Natl Acad Sci USA 98: 6247-6252.

Duron O, Weill M (2006). Wolbachia infection influences the development of Culex pipiens embryos in incompatible crosses. Heredity 96: 493-500.

Giordano R, Jackson JJ, Robertson HM (1997). The role of Wolbachia bacteria in reproductive incompatibilities and hybrid zones of Diabrotica beetles and Gryllus crickets. Proc Natl Acad Sci USA 94: 11439-11444.

Giordano R, O'Neil SL, Robertson HM (1995). Wolbachia infections and the expression of cytoplasmic incompatibility in Drosophila sechellia and D. mauritiana. Genetics 140: 1307-1317.

Harrison RG (1983). Barriers to gene exchange between closely related cricket species. I. Laboratory hybridization studies. Evolution 37: 245-251.

Harrison RG (1985). Barriers to gene exchange between closely related cricket species. II. Life cycle variation and temporal isolation. Evolution 39: 244-259.
Harrison RG, Bogdanowicz SM (1997). Patterns of variation and linkage disequilibrium in a field cricket hybrid zone. Evolution 51: 493-505.

Harrison RG, Rand DM (1989). Mosaic hybrid zones and the nature of species boundaries. In: Otte D, Endler JA (eds) Speciation and its Consequences. Sinauer: Sunderland, MA. pp 111-133.

Harrison RG, Rand DM, Wheeler WC (1987). Mitochondrial DNA variation in field crickets across a narrow hybrid zone. Mol Biol Evol 4: 144-158.

Hilgenboecker K, Hammerstein P, Schlattmann P, Telschow A, Werren JH (2008). How many species are infected with Wolbachia?-a statistical analysis of current data. FEMS Microbiol Lett 281: 215-220.

Hoerauf A, Nissen-Pahle K, Schmetz C, Henkle-Duhrsen K, Blaxter ML, Büttner DW et al. (1999). Tetracycline therapy targets intracellular bacteria in the filarial nematode Litomosoides sigmodontis and results in filarial infertility. J Clin Invest 103: $11-17$.

Hoerauf A, Volkmann L, Hamelmann C, Adjei O, Autenrieth IB, Fleischer B et al. (2000). Endosymbiotic bacteria in worms as targets for a novel chemotherapy in filariasis. Lancet 355 $1242-1243$.

Hoffmann AA, Clancy D, Duncan J (1996). Naturally-occurring Wolbachia infection in Drosophila simulans that does not cause cytoplasmic incompatibility. Heredity 76: 1-8.

Hurst GD, Jiggins FM, Robinson SJW (2001). What causes inefficient transmission of male-killing Wolbachia in Drosophila? Heredity 87: 220-226.

Hurst GD, Johnson AP, Schulenburg JH, Fuyama Y (2000). Male-killing Wolbachia in Drosophila: a temperaturesensitive trait with a threshold bacterial density. Genetics 156: 699-709.

Hurst GDD, Jiggins FM, Schulenburg JHG, Bertrand D, West SA, Goriacheva II et al. (1999). Male-killing Wolbachia in two species of insect. Proc $R$ Soc Lond B Biol Sci 266: 735-740.

Ikeda H (1970). The cytoplasmically-inherited 'sex-ratio' condition in natural and experimental populations of Drosophila bifasciata. Genetics 65: 311-333.

Ivy TM, Sakaluk SK (2005). Polyandry promotes enhanced offspring survival in decorated crickets. Evolution 59: 152-159.

Jaenike J, Dyer KA, Cornish C, Minhas MS (2006). Asymmetrical reinforcement and Wolbachia infection in Drosophila. PLoS Biol 4: e325.

Jeyaprakash A, Hoy MA (2000). Long PCR improves Wolbachia DNA amplification: wsp sequences found in $76 \%$ of sixtythree arthropod species. Insect Mol Biol 9: 393-405.

Lassy CW, Karr TL (1996). Cytological analysis of fertilization and early embryonic development in incompatible crosses of Drosophila simulans. Mech Dev 57: 47-58.

Mandel MJ, Ross C, Harrison RG (2001). Do Wolbachia infections play a role in unidirectional incompatibilities in a field cricket hybrid zone? Mol Ecol 10: 703-709.

Maroja LS (2008). Barriers to gene exchange in a field cricket hybrid zone. PhD dissertation, Cornell University, Ithaca, NY.

Marshall JL (2004). The Allonemobius-Wolbachia host-endosymbiont system: evidence for rapid speciation and against reproductive isolation driven by cytoplasmic incompatibility. Evolution 58: 2409-2425.

McGraw EA, Merritt DJ, Droller NJ, O'Neill SL (2002). Wolbachia density and virulence attenuation after transfer into a novel host. Proc Natl Acad Sci USA 99: 2918-2923.

Navajas M, Tsagkarakov A, Lagnel J, Perrot-Minnot M-J (2000). Genetic differentiation in Tetranychus urticae (Acari: Tetranychidae): polymorphism, host races or sibling species? Exp Appl Acarol 24: 365-376.

O'Neill SL, Giordano R, Colbert AME, Karr TL, Robertson HM (1992). 16S rRNA phylogenetic analysis of the bacterial 
endosymbionts associated with cytoplasmic incompatibility in insects. Proc Natl Acad Sci USA 89: 2699-2702.

O'Neill SO, Karr TL (1990). Bidirectional cytoplasmic incompatibility between conspecific populations of Drosophila simulans. Nature 348: 178-180.

Poinsot D, Bourtzis K, Markakis G, Savakis C, Merlot H (1998). Wolbachia transfer from Drosophila melanogaster into D. simulans: host effect and cytoplasmic incompatibility relationships. Genetics 150: 227-237.

R Development Core Team (2006). R: a language and environment for statistical computing. Vienna, Austria (http://www. R-project.org).

Riparbelli MG, Giordano R, Callaini G (2007). Effects of Wolbachia on sperm maturation and architecture in Drosophila simulans Riverside. Mech Dev 124: 699-714.

Ross CL, Harrison RG (2002). A fine-scale spatial analysis og the mosaic hybrid zone between Gryllus firmus and Gryllus pennsylvanicus. Evolution 56: 2296-2312.

Ross CL, Harrison RG (2006). Viability selection on overwintering eggs in a field cricket mosaic hybrid zone. Oikos 115: 53-68.

Rousset F, Bouchon D, Pintureau B, Juchault P, Solignac M (1992). Wolbachia endosymbionts responsible for various alterations of sexuality in arthropods. Proc $R$ Soc Lond B Biol Sci 250: 91-98.

Sakaluk SK, Schaus JM, Eggert AK, Snedden WA, Brady PL (2002). Polyandry and fitness of offspring reared under varying nutritional stress in decorated crickets. Evolution 56: 1999-2007.

Shoemaker DD, Katju V, Jaenike J (1999). Wolbachia and the evolution of reproductive isolation between Drosophila recens and Drosophila subquinaria. Evolution 53: 1157-1164.

Simmons LW (1988). The calling song of the field cricket Gryllus bimaculatus (De Geer): constraints on transmission and its role in intermale competition and female choice. Anim Behav 36: 380-394.

Stouthamer R, Breeuwer JAJ, Hurst DD (1999). Wolbachia Pipientis: microbial manipulator of arthropod reproduction. Ann Rev Microbiol 53: 71-102.

Stouthamer R, Breeuwer JAJ, Luck RF, Werren JH (1993). Molecular identification of microorganisms associated with parthenogenesis. Nature 361: 66-68.

Telschow A, Hammerstein P, Werren JH (2005a). The effect of Wolbachia versus genetic incompatibilities on reinforcement and speciation. Evolution 59: 1607-1619.
Telschow A, Yamamura N, Werren JH (2005b). Bidirectional cytoplasmic incompatibility and the stable coexistence of two Wolbachia strains in parapatric host populations. J Theor Biol 235: 265-274.

Tram U, Sullivan W (2002). Role of delayed nuclear envelope breakdown and mitosis in Wolbachia-induced cytoplasmic incompatibility. Science 296: 1124-1126.

Vala F, Breeuwer JA, Sabelis MW (2000). Wolbachia induced 'hybrid breakdown' in the two-spotted spider mite Tetranychus urticae Koch. Proc R Soc Lond B Biol Sci 267: 1931-1937.

Veneti Z, Clark ME, Zabalou S, Karr TL, Savakis C, Bourtzis K (2003). Cytoplasmic incompatibility and sperm cyst infection in different Drosophila-Wolbachia associations. Genetics 164: $545-552$

Wagner WE, Kelley RJ, Tucker KR, Harper CJ (2001). Females receive a life-span benefit from male ejaculates in a field cricket. Evolution 55: 994-1001.

Weeks AR, Reynolds KT, Hoffmann AA (2002). Wolbachia dynamics and host effects: what has (and has not) been demonstrated? Trends Ecol Evol 17: 257-262.

Weeks AR, Turelli M, Harcombe WR, Reynolds KT, Hoffmann AA (2007). From parasite to mutualist: rapid evolution of Wolbachia in natural populations of Drosophila. PLoS Biol 5: e114.

Werren JH (1997a). Biology of Wolbachia. Annu Rev Entomol 42: $587-609$.

Werren JH (1997b). Wolbachia and speciation. In: Howard D, Berlocher S (eds). Endless Species and Speciation. Oxford University Press: New York.

Werren JH, Windsor D, Guo RL (1995). Distribution of Wolbachia among neotropical arthropods. Proc Royal Soc Lond B 262: 197-204.

Werren JH, Windsor DM (2000). Wolbachia infection frequencies in insects: evidence of a global equilibrium? Proc $R$ Soc Lond $B$ Biol Sci 267: 1277-1285.

West SA, Cook JM, Werren JH, Godfray HC (1998). Wolbachia in two insect host-parasitoid communities. Mol Ecol 7: 1457-1465.

Willett C, Ford MJ, Harrison RG (1997). Inferences about the origin of a field cricket hybrid zone from a mitochondrial DNA phylogeny. Heredity 79: 484-494.

Yen JH, Barr AR (1971). New hypothesis of the cause of cytoplasmic incompatibility in Culex pipiens. Nature 232: 657-658.

Zchori-Fein E, Gottlieb Y, Coll M (2000). Wolbachia density and host fitness components in Muscidifurax uniraptor (Hymenoptera: pteromalidae). J Invertebr Pathol 75: 267-272. 